\title{
A spatial decision support system for radar-meteorology data in South Africa
}

Peter Löwe*

- Department of Geography, University of Würzburg, Am Hubland, D 97082 Würzburg, Germany, tel. ++49-931-8885585, e-mail loewe@informatik.uni-wuerzburg.de

\section{Introduction}

The South African Weather Service (SAWS) operates a nationwide network of ground-based meteorologic radar stations (National Weather Radar Network), providing data continuity in some regions dating back to 1994, which is unique for southern africa.

The incoming data is processed and archived using the TITAN software package [1]. Thematic digital maps are derived which can be accessed through the METSYS internet presence [2].

During the course of a soil erosion assesment study [3] GRASS was introduced to the former SAWS radar research center (now: METSYS) in 1999. It was realized that a general purpose geographic information system can offer significant advantages over a specialized radar data management system for creating valueadded products for the customers.

GRASS is still used for meteorologic research by METSYS today and remains the GIS of choice because it being FreeSoftware and linux-based.

\section{Use of radar data}

The import of three dimensional reflectivity radar data by ASCII files into the GIS proved unsatisfactory as it created a bottleneck. So an import-tool for TITANgenerated meteorologic data volumes (MDV) was created to access the data in GRASS and to enable quasi-realtime processing of the incoming data stream from the receiving stations: The TITAN environment creates a national dataset every five minutes, thereby allowing "now-casting" services.

A PostgreSQL database system is used to store the radar data fields, quicklooks and meta data for easy access, thereby allowing for the first time multitemporal queries for researchers lacking access to the TITAN system.

\section{Value added products}

The predominantly convective nature of precipitation in southern africa creates a public demand for severe weather information systems for decision support. Such a system was set up in GRASS (on the created data-processing infrastructure), 
using a rule based expert system for precipitation, cloud and storm cell classification.

Storm cells are isolated from the three dimensional reflectivity fields and information about their stages of development are derived. This is achieved by using meteorologic expert knowledge to classify the cells by their reflectivity values.

The thematic data is archived and can be made available to the interested public by electronic mail or short message services (SMS) for areas of interest, e.g. weather hazards warnings.

Further, interactive HTML-maps for the whole nation and its provinces are automatically created after the arrival of each new data volume, which can be used as a front-end for a web-based weather information system[4].

For customers in remote locations this service can also be made accessible by broadcasting the datastream via satellite through the worldspace digital radio system[5].

\section{Expert Systems}

In the course of the development of the knowledge based component were several manifestations of expert systems evaluated:

First, the expert system shell toolkit D3 [6] was used so set up meteorologic knowledge bases and to introduce the METSYS staff to artificial intelligence. Unfortunately, the integration of D3 into the GRASS environment did not seem reasonable at its current level of maturity. Classification algorithms for radar-data (SSS[7], CONSTRAT[8] and Rainrate) were encoded as D3-knowledge bases.

Once the D3-based development had reached its end, these knowledge bases were used as a starting point to reimplement the contained expert knowledge by relying solely on GRASS' built-in inference engine r.infer for the task.

This proved to be non-trivial, as r.infer is still exclusively integer-based and offers only most basic options for regular expresions.

It was soon realized that the combined use of r.mapcalc for the derivation of results from regular expression and r.infer for the final inference is a viable way to implement rule-based algorithms in a GRASS environment without relying on add-on software.

Since the necessary transformation of all floating-point values into integers and the sequential calling-up of script based inference runs appeared awkward and counterintuitive in regard to the targeted meteorologic user group, a more elegant alternative was sought for.

This was finally implemented by using of the CAPE project [9]: CAPE is a version of the CLIPS expert system shell toolkit[10] with an extension for Perl. The extended functionality is used to establish a connection between the GRASS modules and the CAPE-environment. By running CAPE within a GRASS shell and using hybrid CAPE-GRASS-scripts ("GRASSCAPE")[11] as interface modules it is possible to tap the full potential of rule-based and object-orientated programming for spatial queries and vice versa: Entities in CLIPS can query 
spatial information during runtime and can transfer the infered contents back into site, raster or vector-layers.

This approach provides intentionally a high level bridge unlike existing low-level GRASS/CLIPS-connections: It allows to explore rule-based spatial decision systems by using the CAPEs listener/shell for entering GRASS-commands as well, encouraging explorative science by extending the "workbench" already provided by GRASS by a strong artificial intelligence component.

Alternatively, the GRASS-CAPE link can also be used for automated tasks, as CAPE can be called up in a script-run mode. This mode is used to run radarmeteorology knowledge bases, whose design is much more elegant and humanreadible than their r.infer counterparts.

Currently, there is a trade-of between elegance and speed: Running a CAPE-based spatial classification takes up to seven times longer than the same query by r.infer. The bottleneck was indentified as the transformation of spatial data into CLIPS "facts", which will be improved by refactoring these scripts.

\section{Conclusion}

It has been demonstrated that GRASS-based applications can perform well for radar-meteorologic tasks. This is particularly beneficial for developing nations with financial constraints on their research programs.

The algorithms used to infer information about storm cells and precipitation from radar reflectivity fields can be encoded as knowledge bases for rule-based expert systems.

By introducing an interface between GRASS and CAPE a sophisticated expert system shell toolkit can be used within the GRASS GIS to infer thematic maps from such knowledge bases. Such thematic maps can be used for interactive web presences or just-in-time/just-in-place customer notification of weather hazards.

\section{References}

[1] Dixon, M and Wiender G. TITAN: Thunderstorm Identification, Tracking, Analysis and Nowcasting - a Radar-based Methodology. In J.Atmos. Ocean. Tech 10(6), pages $785-797,1993$

[2] http://metsys.weathersa.co.za

[3] Seuffert 0. et al., REI An integrated Rainfall Erositivity Index for assessing and correlating rainfall structure, runoff and erosion. In Geo-Öko-Dynamik, XX,1/2-99, pages 1-54, 1999

[4] http://ki.informatik.uni-wuerzburg.de/ loewe/grass/htmlsachen/frames.html

[5] http://www.worldspace.com

[6] http://d3.informatik.uni-wuerzburg.de 
[7] Visser, P.M. The Storm-Structure-Severity method for the identification of convective storm characteristics with conventional weather radar. In Meteorol. Appl. 8, 1-10, 2001.

[8] Mittermaier, M. Investigating the characteristics of the radar vertical reflectivity profile. Masters Thesis, Department of Civil Engineering, University of Natal, South Africa, 1999.

[9] http://cape.sourceforge.net

[10] http://www.ghg.net/clips/CLIPS.html

[11] http://grasscape.sourceforge.net 